Research Article

\title{
Dynamic Behaviors and Mechanisms of Permanent Magnet Synchronous Motor with Excitation
}

\author{
Shaohua Liu $\mathbb{D}^{1}$, Tingting Shao, ${ }^{1}$ Hai-dong Wu, ${ }^{1}$ Dong-hui Zhang, ${ }^{1}$ and Qing-zhen Han ${ }^{2}$ \\ ${ }^{1}$ School of Automobile Engineering, Changzhou Vocational Institute of Mechatronic Technology, Changzhou 213164, \\ Jiangsu, China \\ ${ }^{2}$ School of Mechanical Engineering, Yangzhou University, Yangzhou 225009, Jiangsu, China \\ Correspondence should be addressed to Shaohua Liu; 1sh1957@czimt.edu.cn
}

Received 5 February 2021; Revised 13 April 2021; Accepted 10 May 2021; Published 4 June 2021

Academic Editor: Massimiliano Ferrara

Copyright ( $\odot 2021$ Shaohua Liu et al. This is an open access article distributed under the Creative Commons Attribution License, which permits unrestricted use, distribution, and reproduction in any medium, provided the original work is properly cited.

\begin{abstract}
The aim of this study is to make a general exploration of the dynamic characteristics of the permanent magnet synchronous motor (PMSM) with parametric or external perturbation. The pitchfork, fold, and Hopf bifurcations are derived by using bifurcation theory. Simulation results not only confirm the theoretical analysis results but also show the Bogdanov-Takens bifurcation of the equilibrium. Dynamic behaviors, such as period three and chaotic motion of PMSM, are analyzed by using bifurcation diagram and phase portraits. The symmetric fold/fold bursting oscillation as well as two kinds of delayed pitchfork bursting oscillations is obtained, and different mechanisms are presented.
\end{abstract}

\section{Introduction}

Since the permanent magnet synchronous motor (PMSM) has low inertia, low noise, high power density, and high efficiency, it is one of most important development trends in a variety of driving motors [1-3]. PMSM is a multivariable, strongly nonlinear, and strongly coupled electromechanical system which is widely used in industry and may exhibit different dynamic behaviors as the parameters and the external load are not fixed but may fluctuate within a range according to the working environment. So, the dynamic behaviors of the PMSM with parametric perturbation as well as with external perturbation should be discussed deeply to provide theoretical basis.

Scholars have done a lot of research on the dynamic characteristics of PMSM. Li et al. [4] derived a dynamic model of PMSM by applying an affine transformation and a time-scaling transformation and studied the complicated dynamic behaviors such as limit cycle and chaos in the smooth air gap PMSM. Jing et al. [5] studied the dynamics of nonsmooth air gap PMSM and derived the bifurcation conditions of pitchfork bifurcation and Hopf bifurcation by using bifurcation theory and center manifold theory. The dynamic behaviors such as the period doubling bifurcation, cyclic fold bifurcation, and chaotic motion are obtained by using numerical simulation. Rasoolzadeh and Tavazoei [6] discussed the dynamic behaviors of PMSM with external excitation and analyzed the effect of excitation angle-frequency on the dynamic behaviors by using bifurcation diagram. Wang et al. [7] analyzed the dynamic response of PMSM under FOC control and obtained different attractors with the change of single parameter. In reference [8], the parameter region is divided into the chaotic area and nonchaotic area by a lot of simulation results, and the maximum torque output of the stable region is estimated based on the nonchaotic area.

Bursting oscillation is a kind of fast-slow dynamic behavior when the dynamic system has two or more time scales [9-12]. At present, codimension one bursting oscillations of the dynamic system are studied deeply in the nervous system. Izhikevich [13] summarized the codimension one bursting oscillations that may happened in the nervous system and studied its classification and naming method, and some of them have already been discovered in the nervous system. Bursting oscillation is also studied in other fields. In reference [14], bursting behaviors of a piecewise 
mechanical system with parameter perturbation in stiffness is studied. Wu et al. [15] discussed the chaotic and periodic bursting phenomena in a memristive Wienbridge oscillator. The time-delayed bursting oscillations in a two dimensional oscillator is studied in reference [16]. Makouo and Woafo [12] studied the bursting patterns in Van der Pol oscillators by experimental observation. Han [17] discussed the fold/fold bursting oscillation and fold/ subHopf bursting oscillation in a hyperchaotic Lorenz system. The high speed spiking and bursting oscillations in a long-delayed broadband optoelectronic oscillator is studied in reference [18]. Bi et al. [19] discussed the mechanism of bursting oscillations with different codimensional bifurcations and nonlinear structures. Analysis of the above literature shows that domestic and foreign scholars have done a lot of research on the fast-slow dynamics, but there are still few reports on PMSM which need to be discussed for the deep exploration of the dynamics of PMSM.

This paper gives a general exploration of the dynamic characteristics of PMSM with parametric excitation or load excitation. PMSM parameters are not limited to a special case. The stability conditions and the bifurcation conditions of the equilibrium have been derived. The local dynamic characteristics of the equilibrium points and the global dynamic characteristics of PMSM are discussed by bifurcation set and bifurcation diagram, respectively. The dynamic behaviors at some specific parameters are studied by phase portraits and Poincaré sections, and three kinds of multiple time scale dynamic behaviors are obtained.

\section{Dynamic Model of PMSM}

The dynamic model of PMSM can be described as a threedimensional nonlinear system [20]:

$$
\left\{\begin{array}{l}
\frac{\mathrm{d} i_{d}}{\mathrm{~d} t}=\frac{\left(u_{d}-R_{1} i_{d}+\omega L_{q} i_{q}\right)}{L_{d}}, \\
\frac{\mathrm{d} i_{q}}{\mathrm{~d} t}=\frac{\left(u_{q}-R_{1} i_{q}-\omega L_{d} i_{d}+\omega \psi_{f}\right)}{L_{q}}, \\
\frac{\mathrm{d} \omega}{\mathrm{d} t}=\frac{\left[n_{p} \psi_{f} i_{q}+n_{p}\left(L_{d}-L_{q}\right) i_{d} i_{q}-T_{L}-\beta \omega\right]}{J},
\end{array}\right.
$$

where $i_{d}, i_{q}, \omega$ represent the $d$ and $q$ axis stator current and motor angular speed, respectively; $u_{d}$ and $u_{q}$ are the $d$ - $q$ axis stator voltage components, respectively, $L_{d}$ and $L_{q}$ are the $d-q$ axis stator inductors, respectively, $R_{1}, \psi_{f}, \beta, J, n_{p}$, and $T_{L}$ are the stator winding resistance, the permanentmagnet flux, the viscous damping coefficient, the polar moment of inertia, the number of pole-pairs, and the external load torque, respectively.

By applying an affine transformation and a time-scaling transformation, system (1) can be transformed into the following dimensionless model:

$$
\left\{\begin{array}{l}
\frac{\mathrm{d} \tilde{i}_{d}}{\mathrm{~d} \widetilde{t}}=-\frac{L_{q}}{L_{d}} \widetilde{i}_{d}+\widetilde{\omega} \widetilde{i}_{q}+\widetilde{u}_{d}, \\
\frac{\mathrm{d} \tilde{i}_{q}}{\mathrm{~d} \widetilde{t}}=-\widetilde{i}_{q}-\widetilde{\omega} \widetilde{i}_{d}+\gamma \widetilde{\omega}+\widetilde{u}_{q}, \\
\frac{\mathrm{d} \widetilde{\omega}}{\mathrm{d} \widetilde{t}}=\sigma\left(\widetilde{i}_{q}-\widetilde{\omega}\right)+\widetilde{\varepsilon i}_{d} \widetilde{i}_{q}-\widetilde{T}_{L},
\end{array}\right.
$$

where $\gamma=\left(n_{p} \psi_{f}^{2} / R_{1} \beta\right) ; \quad \sigma=\left(L_{q} \beta / R_{1} J\right) ; \quad \tilde{u}_{q}=\left(n_{p} L_{q} \psi_{f} u_{q} l\right.$ $\left.R_{1}^{2} \beta\right) ; \tilde{u}_{d}=\left(n_{p} L_{q} \psi_{f} u_{d} / R_{1}^{2} \beta\right) ; \varepsilon=\left(L_{q} \beta^{2}\left(L_{d}-L_{q}\right) / L_{d} J n_{p} \psi_{f}^{2}\right)$; and $\widetilde{T}_{L}=\left(L_{q}^{2} T_{L} / R_{1}^{2} J\right)$.

\section{Equilibrium Point and Bifurcations}

3.1. The Inherent Characteristics of the Smooth-Air-Gap PMSM, Namely, $L_{d}=L_{q}, \widetilde{u}_{d}=0, \widetilde{u}_{q}=0$, and $\widetilde{T}_{L}=0$. System (2) can be written as

$$
\left\{\begin{array}{l}
\frac{\mathrm{d} \tilde{i}_{d}}{\mathrm{~d} \tilde{t}}=-\widetilde{i}_{d}+\widetilde{\omega} \tilde{i}_{q} \\
\frac{\mathrm{d} \bar{i}_{q}}{\mathrm{~d} \widetilde{t}}=-\widetilde{i}_{q}-\widetilde{\omega} \tilde{i}_{d}+\gamma \widetilde{\omega} \\
\frac{\mathrm{d} \widetilde{\omega}}{\mathrm{d} \widetilde{t}}=\sigma\left(\widetilde{i}_{q}-\widetilde{\omega}\right)
\end{array}\right.
$$

There are only two combined parameters, $\gamma$ and $\sigma$, in (3), and each parameter represents a group of parameters of the actual PMSM. These two parameters are assumed to be totally independent of each other for further discussing the dynamic characteristics, and the analytical results obtained from the dynamics analysis procedures will encompass a general class of machines rather than being limited to a specific machine with given parameters. The actual dynamic behaviors can be obtained by substituting the actual parameters into $\gamma$ and $\sigma$.

By the analysis of the equilibrium points, $\left(\widetilde{i}_{d_{0}}, \widetilde{i}_{q_{0}}, \widetilde{\omega}_{0}\right)$, of system (3), the equilibrium points equations are obtained:

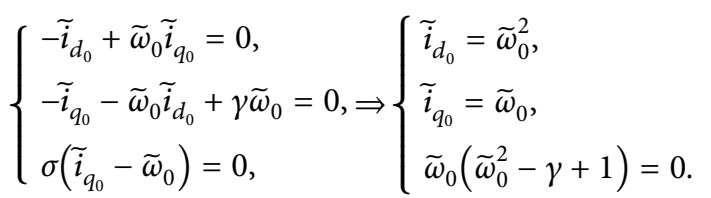

Solving the equilibrium points equations, we obtain the following:

(1) If $\gamma>1$, system (3) would have three equilibrium points, including $(0,0,0),(\gamma-1, \sqrt{\gamma-1}, \sqrt{\gamma-1})$, and $(\gamma-1,-\sqrt{\gamma-1},-\sqrt{\gamma-1})$

(2) If $\gamma \leq 1$, system (3) would only have one equilibrium point, $(0,0,0)$

The local stability of the equilibrium point is determined by the roots of the characteristic equation, and the equilibrium point is stable if the all the roots of the characteristic equation have negative real parts. The characteristic 
equation can be expressed as $\operatorname{det}(\lambda I-J)=0$, where $\lambda, I$, and $J$ represent the eigenvalue, identity matrix, and Jacobian matrix, respectively. In fact, the stability of the equilibrium point is usually judged using Routh-Hurwitz stability criterion $[21,22]$ as solving the characteristic equation is very difficult for high-dimensional dynamic system.

The Jacobian matrix at the equilibrium points of system (3) is

$$
J=\left[\begin{array}{ccc}
-1 & \widetilde{\omega}_{0} & \widetilde{i}_{q_{0}} \\
-\widetilde{\omega}_{0} & -1 & -\widetilde{i}_{d_{0}}+\gamma \\
0 & \sigma & -\sigma
\end{array}\right]
$$

Substituting the Jacobian matrix and equilibrium, $\left(\widetilde{\omega}_{0}^{2}, \widetilde{\omega}_{0}, \widetilde{\omega}_{0}\right)$, into the characteristic equation, $\operatorname{det}(\lambda I-J)=$ 0 , we obtain

$$
\begin{aligned}
\lambda^{3} & +(\sigma+2) \lambda^{2}+\left(1+\sigma \widetilde{\omega}_{0}^{2}-\sigma \gamma+\widetilde{\omega}_{0}^{2}+2 \sigma\right) \lambda \\
& +3 \sigma \widetilde{\omega}_{0}^{2}-\sigma \gamma+\sigma=0 .
\end{aligned}
$$

According to Routh-Hurwitz stability criterion, the sufficient condition of the local stability of the equilibrium points is

$$
\left\{\begin{array}{l}
\sigma>-2 \\
3 \sigma \widetilde{\omega}_{0}^{2}-\gamma \sigma+\sigma>0 \\
(\sigma+2)\left(1+\sigma \widetilde{\omega}_{0}^{2}-\sigma \gamma+\widetilde{\omega}_{0}^{2}+2 \sigma\right)-\left(3 \widetilde{\omega}_{0}^{2}+\gamma-1\right) \sigma>0 .
\end{array}\right.
$$

By the bifurcation theory [23-25] of the equilibrium points, the equilibrium points may lose stability, and the bifurcation behavior occurs when the parameters pass through the key values. In order to obtain the conditions of Hopf bifurcation, setting $\lambda=n j(n \neq 0)$ and substituting it into characteristic equation (6), we obtain

$$
\begin{aligned}
& -n^{3} j-(\sigma+2) n^{2}+\left(1+\sigma \widetilde{\omega}_{0}^{2}-\sigma \gamma+\widetilde{\omega}_{0}^{2}+2 \sigma\right) n j \\
& \quad+3 \sigma \widetilde{\omega}_{0}^{2}-\sigma \gamma+\sigma=0 .
\end{aligned}
$$

Equating real and imaginary parts of the equation,

$$
\left\{\begin{array}{l}
n^{2}=1+\sigma \widetilde{\omega}_{0}^{2}-\sigma \gamma+\widetilde{\omega}_{0}^{2}+2 \sigma, \\
n^{2}=\frac{\left(3 \sigma \widetilde{\omega}_{0}^{2}-\sigma \gamma+\sigma\right)}{(\sigma+2)} .
\end{array}\right.
$$

Then, if Hopf bifurcation occurs at the equilibrium point of (3), the following conditions must be satisfied:

$$
\left\{\begin{array}{l}
\sigma+2>0 \\
1+\sigma \widetilde{\omega}_{0}^{2}-\sigma \gamma+\widetilde{\omega}_{0}^{2}+2 \sigma>0 \\
\left(-\sigma^{2}-2\right) \omega_{0}^{2}+(\gamma-2) \sigma^{2}+(\gamma-4) \sigma-2=0 .
\end{array}\right.
$$

In addition, if $\gamma>1$, system (3) would have two stable equilibrium points and one unstable equilibrium point, and if $\gamma<1$, system (3) would only have one stable equilibrium point, the pitchfork bifurcation occurs at $\gamma=1$.
To verify the theoretical analysis results, the doubleparameter bifurcation curves of system (3) in the $\gamma-\sigma$ plane are obtained by continuation method (see Figure 1), where "BP" and "subH" represent pitchfork bifurcation curve and subcritical Hopf bifurcation curve, respectively. The pitchfork bifurcation curve together with subcritical Hopf bifurcation curve divide the equilibrium parameter plane into three regions, namely, "A," "B," and "C," where the equilibrium has different characteristics in different regions. There is only one stable equilibrium point in " $\mathrm{A}$ " and two stable equilibrium points and one unstable equilibrium point in "B" and three unstable equilibrium points in "C." To verify the above research, Table 1 lists a set of parameters of PMSM [26], and the multiequilibrium coexistence phenomenon (see Figure 2), is obtained by simulation as the parameters of the PMSM are fall in region "B."

3.2. When Considering the External Load Namely, $L_{d}=L_{q}$, $\widetilde{u}_{d}=0, \widetilde{u}_{q}=0$, and $\widetilde{T}_{L} \neq 0$. This is a special case that the control inputs of the system are removed after the motor runs for a period of operation. Let $u=\widetilde{T}_{L}$, then system (2) becomes

$$
\left\{\begin{array}{l}
\frac{\mathrm{d} i_{d}}{\mathrm{~d} \widetilde{t}}=-\widetilde{i}_{d}+\widetilde{\omega} \tilde{i}_{q} \\
\frac{\mathrm{d} \widetilde{i_{q}}}{\mathrm{~d} \widetilde{t}}=-\widetilde{i}_{q}-\widetilde{\omega} \widetilde{i}_{d}+\gamma \widetilde{\omega}, \\
\frac{\mathrm{d} \widetilde{\omega}}{\mathrm{d} \widetilde{t}}=\sigma\left(\widetilde{i}_{q}-\widetilde{\omega}\right)-u .
\end{array}\right.
$$

By analyzing the equilibrium, $\left(\widetilde{i}_{d_{0}}, \widetilde{i}_{q_{0}}, \widetilde{\omega}_{0}\right)$, of system (11), we obtain

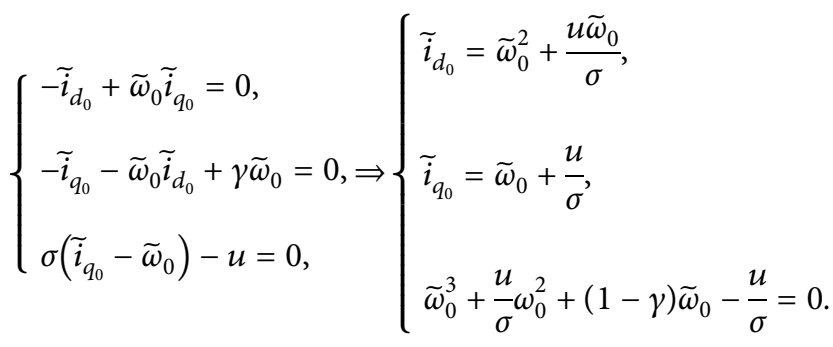

According to the relationship among $\widetilde{i}_{d_{0}}, \widetilde{i}_{q_{0}}, \widetilde{\omega}_{0}$, the equilibrium point of (11) can be written as $\left(\widetilde{\omega}_{0}^{2}+\left(u \widetilde{\omega}_{0} / \sigma\right), \widetilde{\omega}_{0}+(u / \sigma), \widetilde{\omega}_{0}\right)$.

According to the Routh-Hurwitz criteria, the local stability condition of the equilibrium can be written as

$$
\left\{\begin{array}{l}
\sigma+2>0 \\
3 \sigma \widetilde{\omega}_{0}^{2}+u \widetilde{\omega}_{0}+(u-\gamma+1) \sigma>0 \\
\sigma\left(2+2 \sigma+(\sigma+2) \widetilde{\omega}_{0}^{2}+u \widetilde{\omega}_{0}-\gamma(\sigma+1)\right)+2>0 .
\end{array}\right.
$$

If fold bifurcation occurs at the equilibrium of (11), the following conditions must be satisfied: 


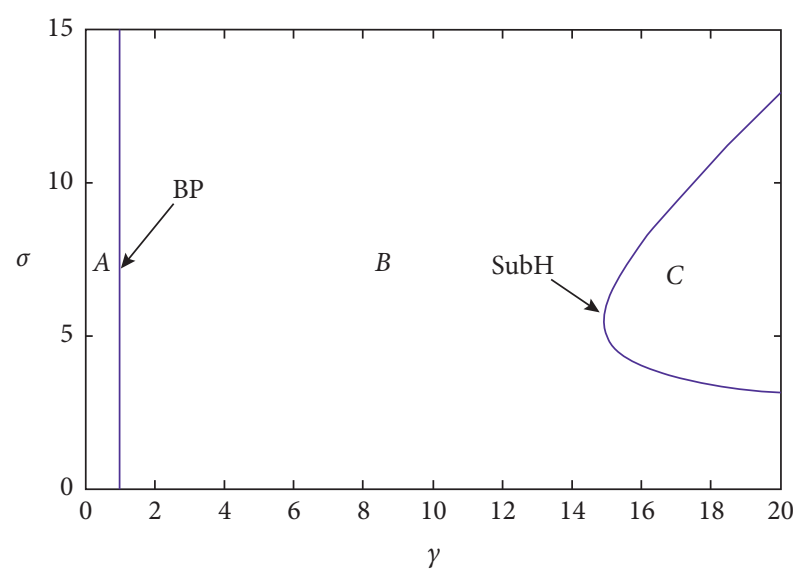

FIgURE 1: Bifurcation set in $\gamma-\sigma$ plane.

TABle 1: Parameters of the PMSM [26].

\begin{tabular}{lcc}
\hline Parameter & Value & Unit \\
\hline$L_{d} / L_{q}$ & 1.01 & $\mathrm{mH}$ \\
$\Psi_{R}$ & 0.06784 & $\mathrm{Nm} / \mathrm{A}$ \\
$R_{1}$ & 0.24 & $\Omega$ \\
$J$ & $4.8 \times 10^{-5}$ & $\mathrm{~kg} \mathrm{~m}$ \\
$p_{n}$ & 4 & \\
$\beta$ & 0.01619 & $\mathrm{~N} / \mathrm{rad} / \mathrm{s}$ \\
\hline
\end{tabular}

$$
\left\{\begin{array}{l}
\widetilde{\omega}_{0}^{3}+\frac{u}{\sigma} \omega_{0}^{2}+(1-\gamma) \widetilde{\omega}_{0}+\frac{u}{\sigma}=0 \\
3 \sigma \widetilde{\omega}_{0}^{2}+u \widetilde{\omega}_{0}+(u-\gamma+1) \sigma=0 .
\end{array}\right.
$$

If Hopf bifurcation occurs at the equilibrium of (11), the following conditions must be satisfied:

$$
\left\{\begin{array}{l}
\sigma+2>0 \\
(1+\sigma) \widetilde{\omega}_{0}^{2}+u \widetilde{\omega}_{0}+(2-\gamma) \sigma+1>0, \\
\widetilde{\omega}_{0}^{3}+\frac{u}{\sigma} \omega_{0}^{2}+(1-\gamma) \widetilde{\omega}_{0}+\frac{u}{\sigma}=0, \\
\sigma\left(2+2 \sigma+(\sigma+2) \widetilde{\omega}_{0}^{2}+u \widetilde{\omega}_{0}-\gamma(\sigma+1)\right)+2=0 .
\end{array}\right.
$$

The bifurcation set of equilibrium of (11) (see Figure 3) describes the bifurcation and stability characteristics of the equilibrium, where the parameter $\gamma$ is fixed as 4.378 obtained by calculating the data from Table 1, and the PMSM itself always has one unstable and two stable equilibrium as shown in Figure 1. "BT" represents the Bogdanov-Takens, one kind of codimension two bifurcation of the equilibrium. The fold bifurcation curve, labelled as "LP," together with subcritical Hopf bifurcation curve, labelled as "subH," divided the equilibrium parameter plane into three regions, namely, " $A$ "- "C." There is only one stable equilibrium in " $A$," and the characteristics of this stable equilibrium are not changed as well as two unstable equilibrium appear when the

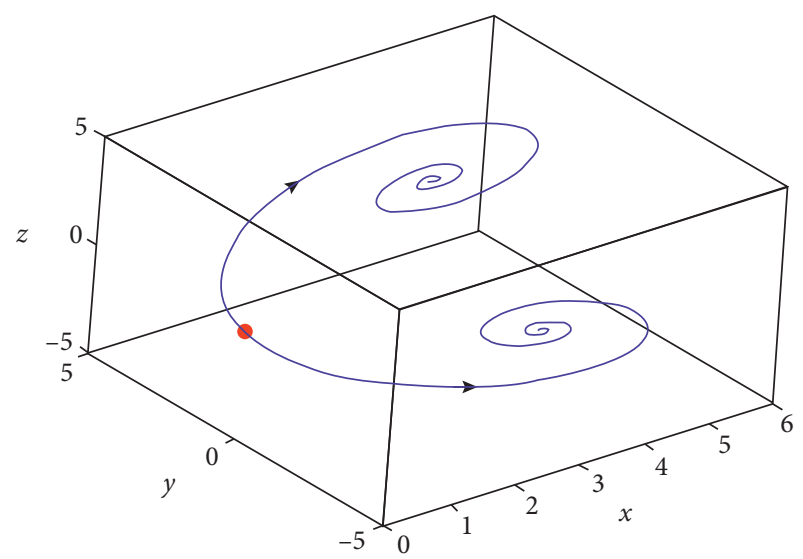

Figure 2: Multiequilibrium coexistence phenomenon.

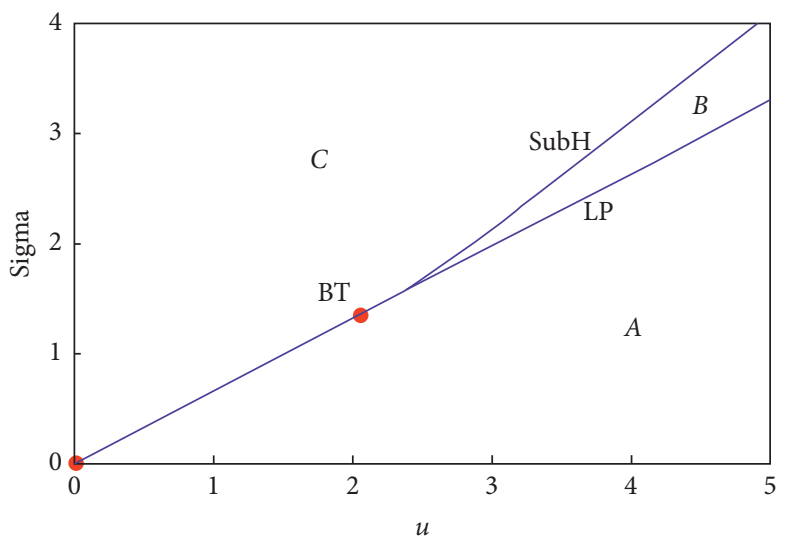

Figure 3: Bifurcation set in the $u-\sigma$ plane.

parameters pass fold bifurcation curve from " $\mathrm{A}$ " to "B." There are one stable and two unstable equilibrium points in "B." When the parameters pass through subcritical Hopf bifurcation line from " $B$ " to " $C$," one of the unstable equilibriums become stable, namely, there are two stable and one unstable equilibrium in "C."

\section{Simulation Results}

The dynamics of the PMSM model with parametric or external excitation will be discussed in this section. Based on the different order of magnitude of the excitation frequency, the dynamic behaviors are divided into two parts. The bifurcation and chaos are discussed in 4.1, and the fast-slow dynamics are discussed in 4.2. For the sake of simplicity, let $x=\widetilde{i}_{d}, y=\widetilde{i}_{q}$, and $z=\widetilde{\omega}$.

4.1. Bifurcation and Chaos. In order to discuss the dynamic characteristics of the PMSM with parametric and external excitation, let $\gamma=\gamma_{0}+A \sin \left(w^{\prime} t\right), u=A \sin \left(w^{\prime} t\right), \quad A=4$, and using the inherent parameters in Table 1 . The bifurcation diagram (see Figure 4) is drawn which describes the global nonlinear dynamic behaviors of the PMSM. The period motion and chaotic motion can be easily identified in 


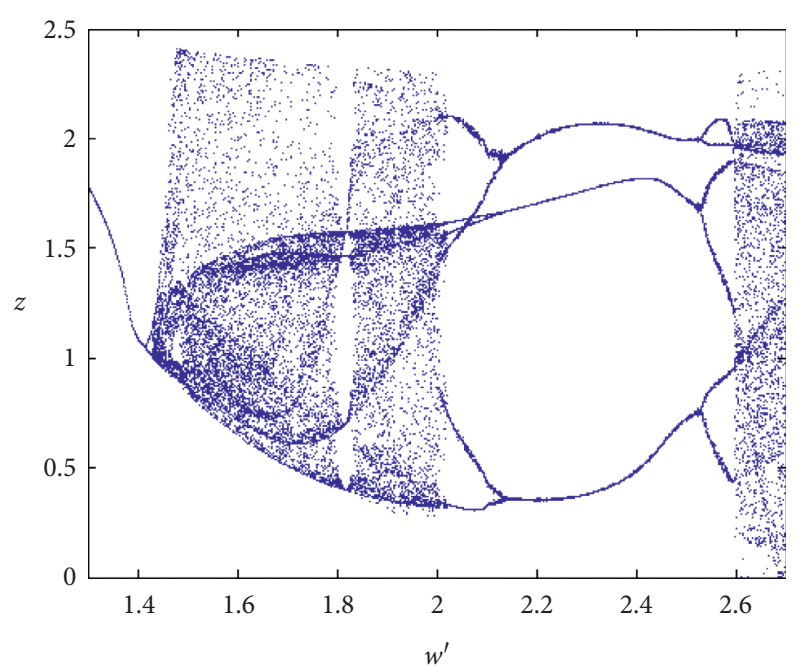

Figure 4: The Poincaré bifurcation diagram.

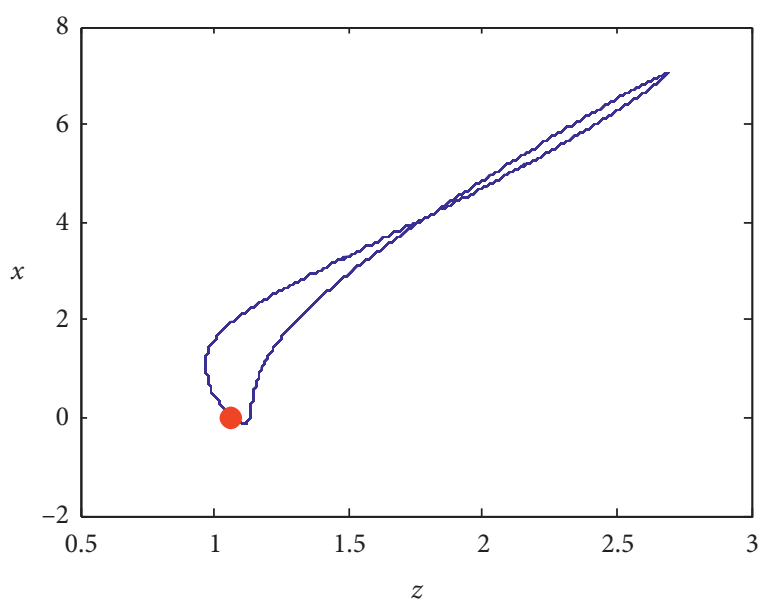

Figure 5: Period one motion, $w^{\prime}=1.41$.

this figure. For further analysis of the dynamic characteristics, the phase portraits and poincaré sections (see Figures 5-8) of different frequencies are drawn where the blue curve and the red dots represent the orbit and the poincaré section, respectively. It can be seen that, with the change of frequency, the PMSM can exhibit different dynamic characteristics, such as the period one motion (see Figure 5), period two motion (see Figure 6), period four motion (see Figure 7), and period three motion (see Figure 8).

4.2. Fast-Slow Dynamics. An interesting two time scale fastslow dynamic [14, 16, 17] behavior (see Figure 9(a)) of PMSM with load excitation is obtained by using the inherent parameters in Table 1 and letting $u=A \sin w^{\prime} t$, and the corresponding time history map is given in Figure 9(b). In order to analyze the mechanism of these phenomena, the equilibrium curve and the overlap of transformed phase portraits (TPP) and equilibrium curve are drawn in Figures $9(\mathrm{c})$ and $9(\mathrm{~d})$, respectively.

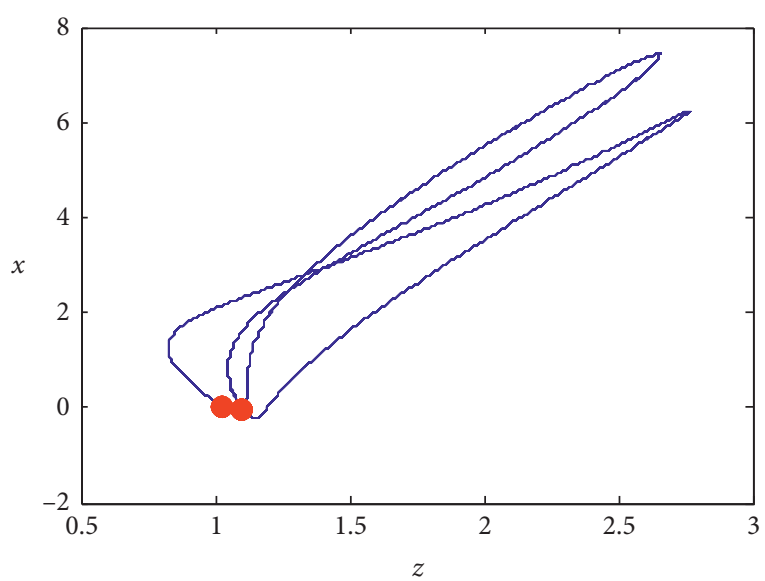

Figure 6: Period two motion, $w^{\prime}=1.425$.

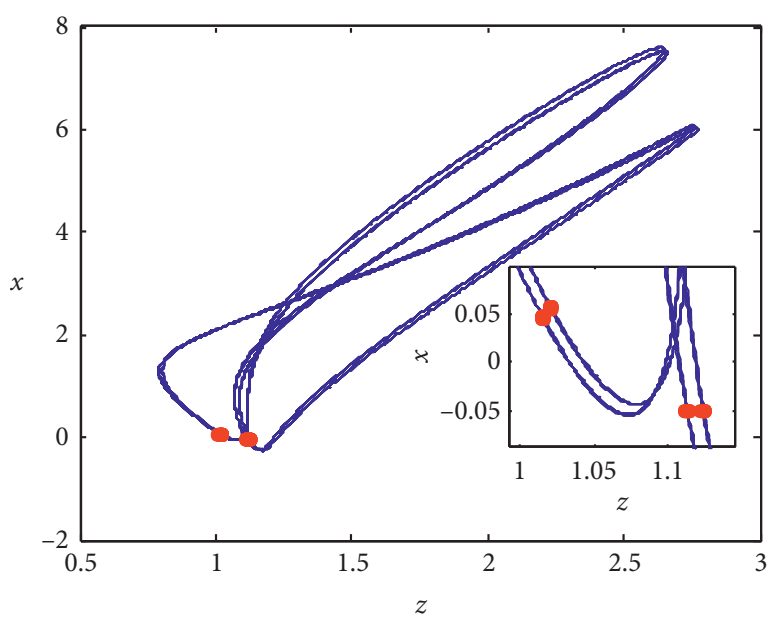

Figure 7: Period four motion, $w^{\prime}=1.429$.

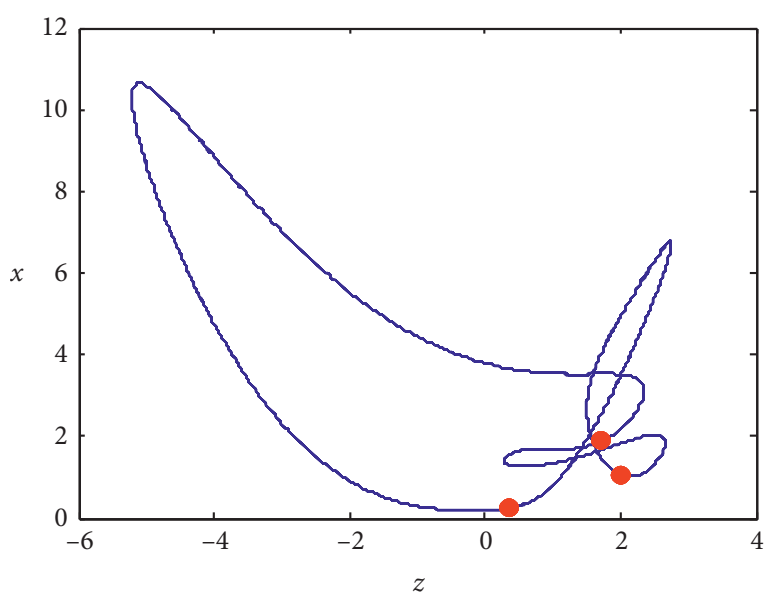

Figure 8: Period three motion, $w^{\prime}=2.195$.

Suppose the trajectory starts from "A" of the up piece of equilibrium curve " $E_{+}$" in Figure $9(d)$, where the red curve is the TPP and the blue curve is the equilibrium curve, and moves strictly until the fold bifurcation point "LP1" and then the trajectory falls into the attraction domain of low piece of 


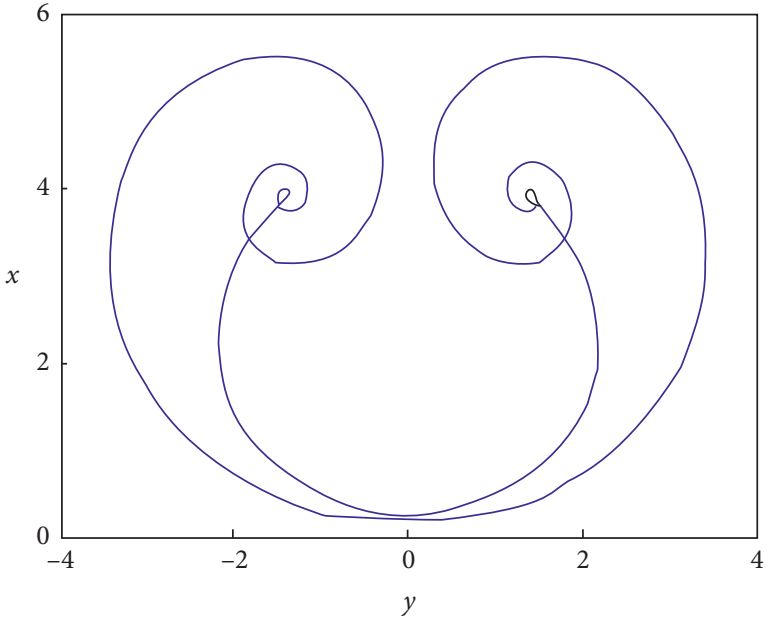

(a)

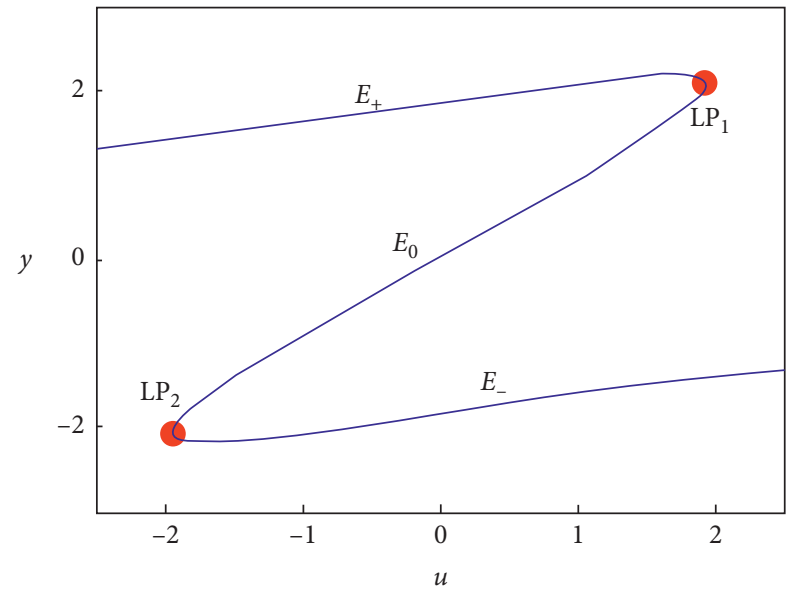

(c)

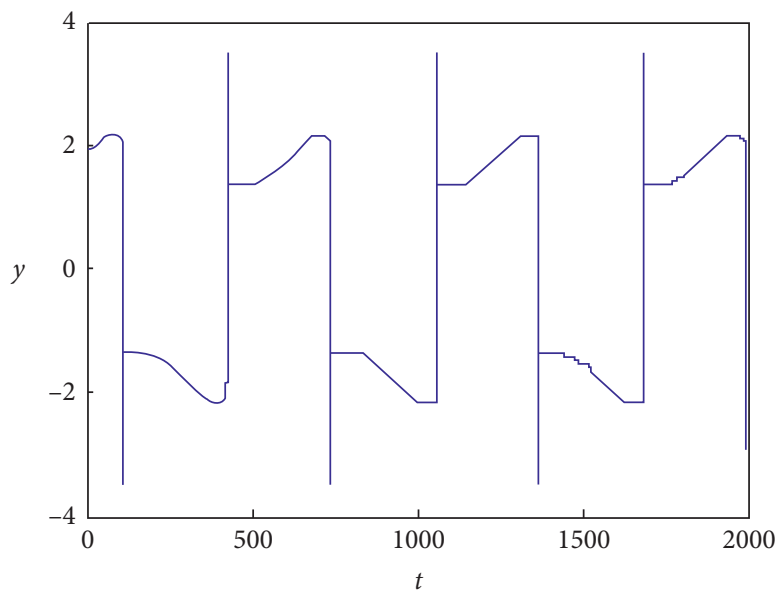

(b)

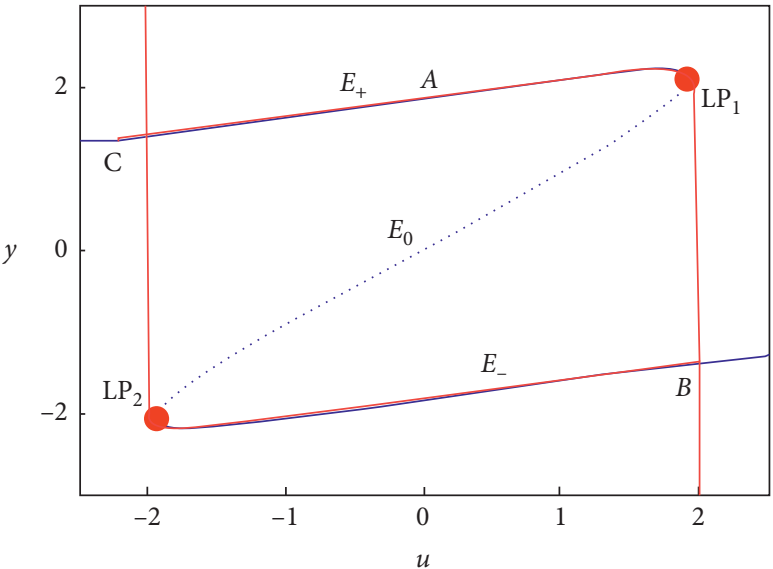

(d)

FIgure 9: Symmetric fold/fold bursting. (a) Phase portrait. (b) Time history. (c) Equilibrium curve. (d) Overlap of TPP and equilibrium curve.

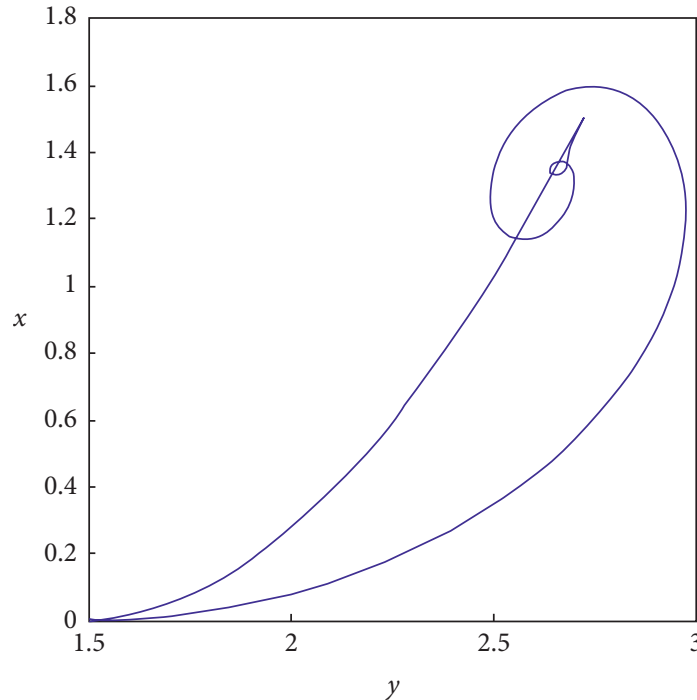

(a)

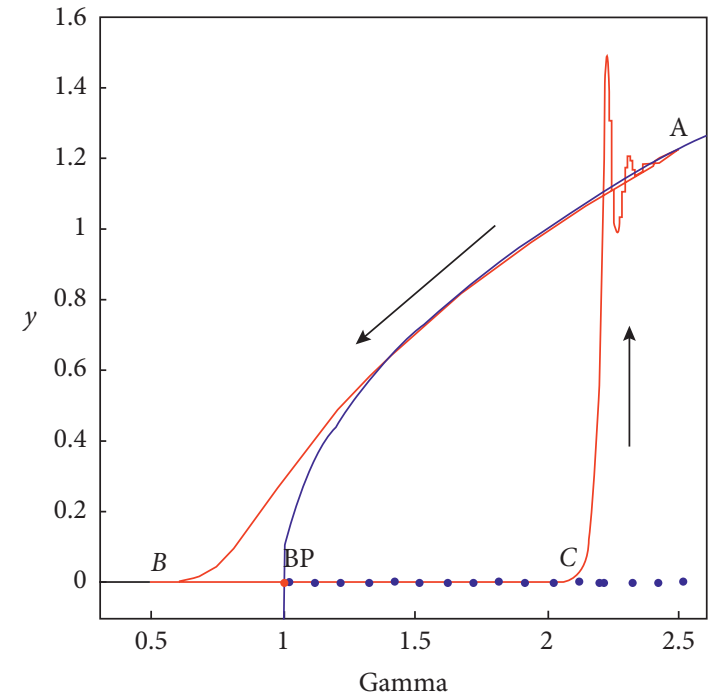

(b)

FIgURE 10: Delayed pitchfork bursting I; $w^{\prime}=0.03, \sigma=10$, and $\gamma=1.5$. (a) Phase portrait. (b) Overlap of TPP and equilibrium curve. 


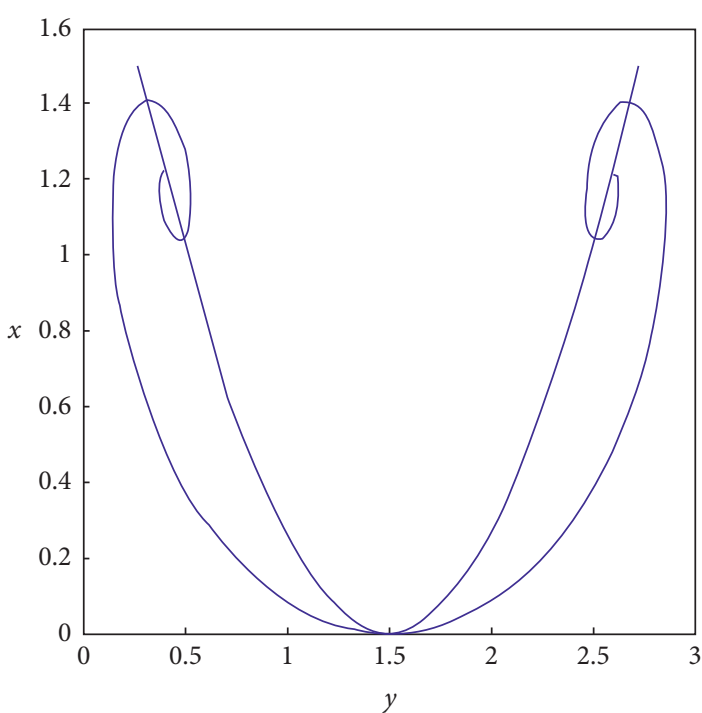

(a)

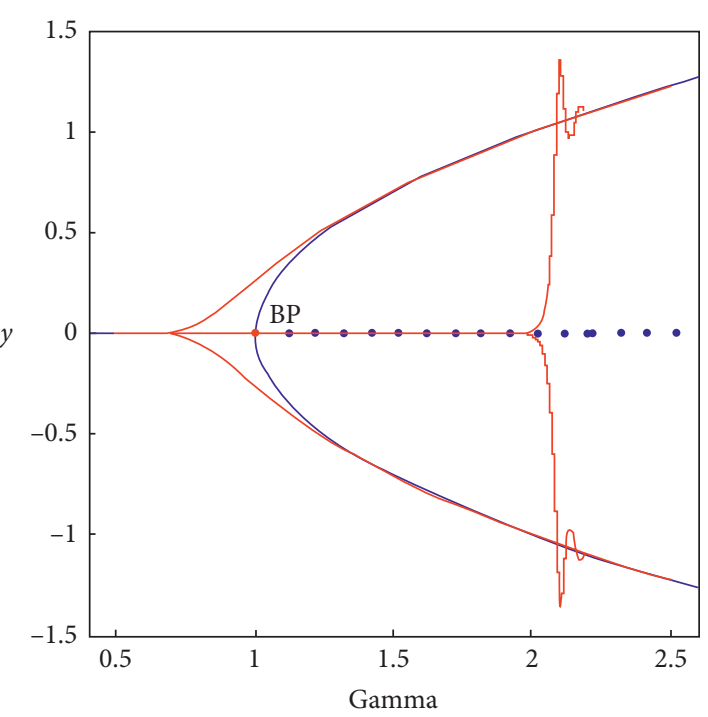

(b)

FIgURE 11: Delayed pitchfork bursting II; $w^{\prime}=0.02, \sigma=10$, and $\gamma_{0}=1.5$. (a) Phase portrait. (b) Overlap of TPP and equilibrium curve.

equilibrium curve " $E_{-}$" as the stable equilibrium curve " $E_{+}$" disappears. Similar dynamics take place at the fold bifurcation point "LP2." A cycle is completed when the trajectory is back to the initial point, and this dynamic behavior is caused by the fold bifurcation of the equilibrium curve and can be named as "symmetric fold/fold bursting."

When considering about the parametric excitation, i.e., $\gamma=\gamma_{0}+A \sin \left(w^{\prime} t\right)$ and $u=0$, two kinds of delayed pitchfork bursting oscillations are obtained (see Figures 10 and 11). In order to analyze the mechanisms of these two kinds of dynamic behaviors, the overlap of TPP and equilibrium curve is drawn in Figures 10(b) and 11(b), where the red and blue curve represent the TPP and equilibrium curve, respectively. For the delayed pitchfork bursting I (see Figure 10(b)), suppose the orbit starts from point " $A$ " and moves to " $\mathrm{B}$ " point as indicated by the arrow, the orbit is not jumped from the up piece of the equilibrium curve to the middle piece of the equilibrium curve at "BP" point because of the delay of pitchfork bifurcation. When the orbit moves from "B" to "C," for the same reason, the orbit is not jumped from the middle piece of the equilibrium curve to the up piece of the equilibrium curve at point "BP," and the orbit is back to point "A" finally. As caused by the delay of pitchfork bifurcation, this dynamic behavior can be named as "delayed pitchfork bursting." For the delayed pitchfork bursting II, the orbit may be attracted by the up piece or the down piece of the equilibrium curve, which is different from the delayed pitchfork bursting I.

\section{Conclusion}

The nonlinear dynamic behaviors of the PMSM with parametric or load perturbation are generally studied. The primary conclusions of this paper are as follows:

(1) Different kinds of bifurcations, such as pitchfork bifurcation, fold bifurcation, and Hopf bifurcation, are deduced. The bifurcation curves are also simulated.

(2) The nonlinear dynamics of the PMSM with both parametric excitation and load excitation are simulated. Different kinds of motions, such as period one, period two, and period four, are obtained. The period three motion is also obtained which can prove the existence of chaotic motion.

(3) When the frequency of the excitation is superlow, the fast-slow dynamics are obtained. Simulation results show three kinds of fast-slow dynamics: symmetric fold/fold bursting oscillation of the PMSM with external perturbation, two kinds of delayed pitchfork bursting oscillations of the PMSM with parametric perturbation. The mechanisms of those three kinds of fast-slow dynamics are also analyzed by using bifurcation curve of the equilibrium.

\section{Data Availability}

The data used to support the findings of this study are included within the article.

\section{Conflicts of Interest}

The authors declare that they have no conflicts of interest.

\section{Acknowledgments}

The authors thank Changzhou Institute of Mechatronic Technology and Jiangsu University for funding this study. The authors thank Jiangsu University Natural Science Foundation Project (16KJB580012) and Overseas Research and Training Program for Young and Middle-Aged Backbone Teachers in Jiangsu Province ((2016)13). 


\section{References}

[1] X. Chen, S. Yuan, and Z. Peng, "Nonlinear vibration for PMSM used in HEV considering mechanical and magnetic coupling effects," Nonlinear Dynamics, vol. 80, no. 1-2, pp. 541-552, 2015.

[2] M. Comanescu, "Speed, rotor position and load torque estimation of the PMSM using an extended dynamic model and cascaded sliding mode observers," in Proceedings of the International Symposium on Power Electronics, Electrical Drives, Automation and Motion, pp. 98-103, IEEE, Capri, Italy, June 2016.

[3] G. Bramerdorfer, W. Amrhein, and S. M. Winkler, "Identification of a nonlinear PMSM model using symbolic regression and its application to current optimization scenarios," in Proceedings of the Industrial Electronics Society IECON 2014 - Conference of the IEEE, pp. 628-633, Dallas, TX, USA, November 2014.

[4] Z. Li, J. B. Park, Y. H. Joo et al., "Bifurcations and chaos in a permanent-magnet synchronous motor," IEEE Transactions on Circuits \& Systems I Fundamental Theory \& Applications, vol. 49, no. 3, pp. 383-387, 2002.

[5] Z. Jing, C. Yu, and G. Chen, "Complex dynamics in a permanent-magnet synchronous motor modelis," Chaos, Solitons \& Fractals, vol. 22, no. 4, pp. 831-848, 2004.

[6] A. Rasoolzadeh and M. S. Tavazoei, "Prediction of chaos in non-salient permanent-magnet synchronous machines," Physics Letters A, vol. 377, no. 1-2, pp. 73-79, 2012.

[7] L. Wang, J. Fan, and Z. Wang, "Dynamic analysis and control of a permanent magnet synchronous motor with external perturbation," Journal of Dynamic Systems Measurement \& Control, vol. 138, no. 1, Article ID 11003, 2015.

[8] S. Wahid, H. Khammari, and M. F. Mimouni, "On some parametric and phase space singularities in PMSM submitted to FOC drive," in Proceedings of the International Conference on Modelling, Identification and Control, Sousse, Tunisia, December 2015.

[9] C. Sourdis, "On periodic orbits in a slow-fast system with normally elliptic slow manifold," Mathematical Methods in the Applied Sciences, vol. 37, no. 2, pp. 270-276, 2014.

[10] X. J. Han, Q. Bi, and P. Ji, "Fast-slow analysis for parametrically and externally excited systems with two slow rationally related excitation frequencies," Physical Review E, vol. 92, no. 1, Article ID 12911, 2015.

[11] M. Farazmand and T. Sapsis, "Dynamical indicators for the prediction of bursting phenomena in high-dimensional systems," Physical Review E, vol. 94, no. 3, Article ID 32212, 2016.

[12] L. Makouo and P. Woafo, "Experimental observation of bursting patterns in Van der Pol oscillators," Chaos, Solitons \& Fractals, vol. 94, pp. 95-101, 2017.

[13] E. M. Izhikevich, "Neural excitability, spiking and bursting," International Journal of Bifurcation and Chaos, vol. 10, no. 6, pp. 1171-1266, 2000.

[14] X. Li and J. Hou, "Bursting phenomenon in a piecewise mechanical system with parameter perturbation in stiffness," International Journal of Non-linear Mechanics, vol. 81, pp. 165-176, 2016.

[15] H. Wu, B. Bao, and Z. Liu, "Chaotic and periodic bursting phenomena in a memristive Wien-bridge oscillator," Nonlinear Dynamics, vol. 83, no. 1-2, pp. 893-903, 2015.

[16] Y. Yu, H. Tang, X. Han, and Q. Bi, "Bursting mechanism in a time-delayed oscillator with slowly varying external forcing," Communications in Nonlinear Science and Numerical Simulation, vol. 19, no. 4, pp. 1175-1184, 2014.
[17] X. J. Han, "Analysis of the fast-slow hyperchaotic Lorenz system," Acta Physica Sinica, vol. 58, no. 9, pp. 6006-6015, 2009.

[18] B. Romeira, F. Kong, J. M. L. Figueiredo, J. Javaloyes, and J. Yao, "High-speed spiking and bursting oscillations in a long-delayed broadband optoelectronic oscillator," Journal of Lightwave Technology, vol. 33, no. 2, pp. 503-510, 2015.

[19] Q. Bi, S. Li, and J. Kurths, "The mechanism of bursting oscillations with different codimensional bifurcations and nonlinear structures," Nonlinear Dynamics, vol. 85, pp. 9931005, 2016.

[20] J. P. Singh, B. K. Roy, and H. V. Kuznetsov, "Multistability and hidden attractors in the dynamics of permanent magnet synchronous motor," International Journal of Bifurcation and Chaos, vol. 4, no. 29, Article ID 1950056, 2019.

[21] A. Pereda, L. A. Vielva, A. Vegas, and A. Prieto, "Analyzing the stability of the FDTD technique by combining the von Neumann method with the Routh-Hurwitz criterion," IEEE Transactions on Microwave Theory and Techniques, vol. 49, no. 2 , pp. $377-381,2001$.

[22] K. T. Alligood, T. D. Sauer, and J. A. York, Chaos: An Introduction to Dynamical Systems, Spinger-Verlag, New York, NY, USA, 1996.

[23] B. D. Hassard, N. D. Kazarinoff, and Y. H. Wan, Theory and Applications of Hopf Bifurcation, Cambridge University Press, Cambridge, UK, 1981.

[24] P. Liu, J. Shi, and Y. Wang, "Imperfect transcritical and pitchfork bifurcations," Journal of Functional Analysis, vol. 251, no. 2, pp. 573-600, 2007.

[25] B. Fiedler, S. Yanchuk, and V. Flunkert, "Delay stabilization of rotating waves near fold bifurcation and application to alloptical control of a semiconductor laser," Physical Review E, vol. 77, no. 2, pp. 601-611, 2008.

[26] Z. Deng, J. Shang, and X. Nian, "Synchronization controller design of two coupling permanent magnet synchronous motors system with nonlinear constraints," Isa Transactions, vol. 59, pp. 243-255, 2015. 